(1)

CrossMark

\title{
It's time to evolve from Scadding: phenotyping sarcoidosis
}

\author{
Daniel A. Culver ${ }^{1}$ and Robert P. Baughman ${ }^{2}$ \\ Affiliations: 'Dept of Pulmonary Medicine, Cleveland Clinic, Cleveland, OH, USA. ${ }^{2}$ University of Cincinnati, \\ Cincinnati, $\mathrm{OH}, \mathrm{USA}$. \\ Correspondence: Daniel A. Culver, Dept of Pulmonary Medicine, Cleveland Clinic, 9500 Euclid Avenue, \\ Cleveland, OH 44195, USA. E-mail: culverdaccf.org
}

@ERSpublications

Novel approaches to cluster sarcoidosis by organ clusters may yield new clinical and pathophysiologic insights http://ow.ly/uCVG30hMsro

Cite this article as: Culver DA, Baughman RP. It's time to evolve from Scadding: phenotyping sarcoidosis. Eur Respir J 2018; 51: 1800050 [https://doi.org/10.1183/13993003.00050-2018].

Tell me with whom you associate, and I will tell you who you are-Johann Wolfgang von Goethe

Prediction is very difficult, especially about the future-Niels Bohr

Sarcoidosis is complex and highly variable, with protean clinical manifestations and a wide array of consequences for patients. The course is likewise unpredictable, leading to the moniker "sarcoidoses" to connote that sarcoidosis may be a syndrome rather than a single disease [1]. Delineating distinct subgroups, "phenotypes", has been an attempt to simplify prediction about individual patients and to homogenise groups for research purposes. Sarcoidosis phenotypes have been used most often to predict prognosis or to cluster patients with similar outcomes [2-5]. But phenotyping could also be used primarily to stratify patients by clinical features such as extent of organ involvement, or by perceived severity $[6,7]$.

The most well-known phenotyping system was proposed by Karl Wurm [8], and modified by Guy Scadding in a seminal 1961 publication [9]. The Wurm-Scadding system relies entirely on the chest radiograph (CXR) to provide a rough estimate of the likelihood of resolution at 5 years. Today, nearly all clinical and translational studies contain a table describing the CXR stage of their population; newer proposed phenotyping systems [4,5] and almost all extant clinical trials likewise incorporate the Wurm-Scadding features as foundational elements. That the Scadding system has persisted so long is a testament to its simplicity, convenience and reproducibility across populations. However, Scadding himself noted from the outset that the likelihood of ocular or cutaneous disease bore no relationship to the chest radiographic pattern [9]. In fact, the Scadding stage correlates poorly with patient-reported disease severity, pulmonary function tests, and need for treatment for individual patients [10-13].

Many of the most reproducible scientific observations regarding sarcoidosis, and a significant proportion of novel pathophysiologic insights, have been derived from focusing on narrow, relatively homogeneous, and fairly uncommon, phenotypes, especially Löfgren's syndrome [14-16]. More recently, a distinct

Received: Jan 092018 | Accepted: Jan 112018

Conflict of interest: D.A. Culver has received non-financial support from Araim and Gilead, and grants and personal fees from Mallinckrodt and Foundation for Sarcoidosis Research, outside the submitted work. R.P. Baughman has received grants from Gilead, Bayer, National Institutes of Health, Genentech, Mallinckrodt and Foundation for Sarcoidosis Research, personal fees for consultancy from Genentech and Mallinckrodt, and fees for speaking from Mallinckrodt, outside the submitted work.

Copyright CERS 2018 
phenotype of advanced sarcoidosis, characterised by markedly impaired physiology, fibrosis and a high prevalence of pulmonary hypertension, was shown to have a particularly poor prognosis [17, 18]. Advanced fibrotic sarcoidosis with pulmonary hypertension and Löfgren's syndrome occupy opposite ends of the spectrum, but for most pulmonary patients, defining a homogeneous and highly predictable phenotype is not so simple. The Scadding staging system is not especially useful for this middle group.

Time and technology have also eroded the usefulness of the Scadding system. Today, nearly all sarcoidosis patients have had at least one computed tomography (CT) study prior to diagnosis. Typically, the scans reveal imaging abnormalities not evident on CXR, including prominent lymph nodes, subtle parenchymal involvement, or minimal fibrosis. The implications of such findings in the context of Scadding descriptions are largely unknown, but it is likely that CT features can enrich our capacity to phenotype $[19,20]$. FDG-PET scan provides further data regarding disease activity and prognosis [21], and will increasingly be considered as a strategy to stratify patients. Other imaging technologies will emerge [2123]. Finally, the field of sarcoidosis is rapidly expanding from being solely the bailiwick of chest physicians to intense focus by other specialties, especially cardiology, rheumatology, ophthalmology and neurology. A phenotyping system centred on the lung is increasingly less relevant.

The GenPhenReSa consortium is an ambitious multinational collaboration that illuminates a new paradigm for sarcoidosis research by demonstrating the feasibility and value of funding collaborative multicentre efforts for this rare and heterogeneous disease. For a study published in this issue of the European Respiratory Journal, the investigators have mined the clinical data from 2163 patients recruited at 31 centers across Central and Western Europe [24]. Rather than binning their patients according to preconceived or historically studied subgroups, the authors performed unsupervised hierarchical clustering on principal components to identify novel clinical associations. Five phenotypic organ-based clusters emerged from the analysis: pulmonary, intra-abdominal organs, musculoskeletal-cutaneous, extrapulmonary and oculo-cardiac-central nervous system-cutaneous (OCCC). Membership in a given cluster entails higher odds for certain other clinical features, such as acute versus subacute onset, symptoms and need for therapy.

The organ clusters revealed in this analysis accord with our existing knowledge. Splenic, hepatic and cutaneous involvement, along with a high frequency of constitutional symptoms, correlated more often than controls in a cohort of 233 US patients [25]. In the Sarcoidosis Genetic Analysis (SAGA) study in African-Americans, liver, spleen and bone marrow involvement segregated into a phenotypic cluster [26]. Other groups have noted a relationship between neurological, cardiac and ocular sarcoidosis [27, 28]. The symptoms and organ distribution of the musculoskeletal-cutaneous phenotype correspond well with Löfgren's syndrome.

While these results are tantalising, they will require validation. A high proportion $(42 \%)$ of the patients were enrolled from a single centre. The relative frequency and importance of acute sarcoidosis in Europe compared with other continents likely skewed the derivation of the clusters. Compared with white Europeans, well-known phenotypic differences are present in African-American and Japanese populations [29-31]. However, besides epidemiological cohorts, analysis of genetic and genomic datasets may provide additional validation through the process of "reverse phenotyping" that could substantiate the currently proposed organ clusters [32,33].

Clearly, clustering patients according to organ involvement will not entirely explain the phenotypic heterogeneity that characterises the sarcoidosis syndrome. However, it is intriguing that organ involvement clusters associate with shared genetic markers [16, 26], at least in selected populations. Shared pathophysiology may also have shared therapeutic implications: for example, the OCCC system contains most of the organs that are most effectively treated with tumour necrosis factor antagonists [34]. We can imagine that biomarker discovery will be enriched when organ clusters are used to analyse candidate proteins, micro-RNAs or genes. There is good precedent for this approach. For example, genetic linkage in the SAGA cohort was stronger for organ involvement when organs were considered as clusters, rather than individually [26].

As mentioned previously, one of the important utilities of phenotyping is to estimate prognosis. To date, phenotyping provides limited insight regarding prognosis. For example, most patients who die from pulmonary sarcoidosis have Scadding stage 4 disease. However, the majority of stage 4 patients still do well over time [18]. The GenPhenReSa consortium did not provide nuanced information about types of therapy and aggressiveness of treatment, and also lacks information about the clinical status at two to five years. Linking clinical outcome to phenotype may lead to future insights, such as genetic markers. For Löfgren's syndrome, between 10 to $20 \%$ of patients require therapy beyond 2 years. Longitudinal studies of Löfgren's patients demonstrated that the patients with HLA type $\mathrm{DRB} 1^{\star} 03$ had self-limited disease whereas half of $\mathrm{DRB}^{\star} 03$ negative patients did not resolve within 2 years [15]. Therefore future 
phenotyping studies of sarcoidosis should plan on following clinical course to further refine the phenotypes identified.

A phenotype is defined as a "set of observable characteristics", but does not dictate the stringency (intensity and/or frequency) with which attempts to uncover any given characteristic should be undertaken [35]. In clinical practice, we phenotype based on what is observed, not what could be observed. This nuance has direct relevance to sarcoidosis, where it is well-known that occult, clinically non-bothersome organ involvement is very common. If one looks hard, perhaps by imaging, random biopsy, or even autopsy, asymptomatic organ involvement may be found much more often than expected [36-40]. For this reason, certain tests have been recommended as baseline screening for all patients, even when asymptomatic [41-43], when occult involvement is potentially organ-threatening. Ophthalmologic examination is one example. However, the intensity of screening, considering cost, radiation exposure, likelihood of changing the outcome, and the subjecting the patient to unnecessary tests or additional downstream procedures, is still a controversial topic $[35,41]$. For example, it is worth noting that the chance of developing new ocular involvement over the first 2 years in a US cohort was 3.3\% [44], and zero in a Japanese cohort over a median follow-up period of 7.7 years [45]. For new cardiac disease, the incidence was $2.3 \%$ and $4.7 \%$ in the two cohorts, respectively. In both series, clinical features at baseline, such as extrathoracic disease or multiple organ involvement predicted a higher chance of new organ manifestations. Coupled with organ-involvement clusters proposed in the current report, these observations suggest a strategy to enrich screening efforts for diagnosing unsuspected organ involvement. For example, routine ophthalmologic exams and more intense (baseline and longitudinal) cardiac screening tests seem more sensible in patients with established neurosarcoidosis, especially if there is multisystem disease.

As pathophysiologic insights, clinical technology, cost, treatments, and practice standards evolve, phenotyping will continue to evolve as well. Biomarkers, genotypes, molecular signatures and clinical features are all likely to play a role in the physician's assessment of sarcoidosis in the near future, ideally in combination. A non-biased approach to clustering sarcoidosis patients based on "-omics" promises to identity novel phenotypes that are more powerful for prognosis, severity, treatment response, and other clinical characteristics. As these new phenotypes are developed, they must be interpreted and validated within the context of the sarcoidosis clinic and the patient's experience in order to be acceptable and useful.

\section{References}

1 Culver DA, Newman LS, Kavuru MS. Gene-environment interactions in sarcoidosis: challenge and opportunity. Clin Dermatol 2007; 25: 267-275.

2 Baughman RP, Nagai S, Balter M, et al. Defining the clinical outcome status (COS) in sarcoidosis: results of the WASOG task force. Sarcoidosis Vasc Diffuse Lung Dis 2011; 28: 56-64.

3 Prasse A, Katic C, Germann M, et al. Phenotyping sarcoidosis from a pulmonary perspective. Am J Respir Crit Care Med 2008; 177: 330-336.

4 Moller DR, Koth LL, Maier LA, et al. Rationale and design of the Genomic Research in Alpha-1 Antitrypsin Deficiency and Sarcoidosis (GRADS) study. Sarcoidosis protocol. Ann Am Thorac Soc 2015; 12: 1561-1571.

5 Rodrigues SC, Rocha NA, Lima MS, et al. Factor analysis of sarcoidosis phenotypes at two referral centers in Brazil. Sarcoidosis Vasc Diffuse Lung Dis 2011; 28: 34-43.

$6 \quad$ Wasfi YS, Rose CS, Murphy JR, et al. A new tool to assess sarcoidosis severity. Chest 2006; 129: 1234-1245.

7 Takada K, Ina Y, Noda M, et al. The clinical course and prognosis of patients with severe, moderate or mild sarcoidosis. J Clin Epidemiol 1993; 46: 359-366.

8 Wurm K. [The significance of stage classification of sarcoidosis (Boeck's disease)]. Dtsch Med Wochenschr 1960; 85: 1541-1548.

9 Scadding JG. Prognosis of intrathoracic sarcoidosis in England. A review of 136 cases after five years' observation. Br Med J 1961; 5261: 1165-1172.

10 Baughman RP, Judson MA, Teirstein A, et al. Presenting characteristics as predictors of duration of treatment in sarcoidosis. QJM 2006; 99: 307-315.

11 Judson MA, Gilbert GE, Rodgers JK, et al. The utility of the chest radiograph in diagnosing exacerbations of pulmonary sarcoidosis. Respirology (Carlton, Vic) 2008; 13: 97-102.

12 Karetzky M, McDonough M. Exercise and resting pulmonary function in sarcoidosis. Sarcoidosis Vasc Diffuse Lung Dis 1996; 13: 43-49.

13 Yeager H, Rossman MD, Baughman RP, et al. Pulmonary and psychosocial findings at enrollment in the ACCESS study. Sarcoidosis Vasc Diffuse Lung Dis 2005; 22: 147-153.

14 Kaiser Y, Lepzien R, Kullberg S, et al. Expanded lung T-bet ${ }^{+} \mathrm{ROR} \gamma \mathrm{T}^{+} \mathrm{CD} 4^{+} \mathrm{T}$-cells in sarcoidosis patients with a favourable disease phenotype. Eur Respir J 2016; 48: 484-494.

15 Grunewald J, Eklund A. Lofgren's syndrome: human leukocyte antigen strongly influences the disease course. Am J Respir Crit Care Med 2009; 179: 307-312.

16 Sato H, Woodhead FA, Ahmad T, et al. Sarcoidosis HLA class II genotyping distinguishes differences of clinical phenotype across ethnic groups. Hum Mol Genet 2010; 19: 4100-4111.

17 Walsh SL, Wells AU, Sverzellati $\mathrm{N}$, et al. An integrated clinicoradiological staging system for pulmonary sarcoidosis: a case-cohort study. Lancet Respir Med 2014; 2: 123-130.

18 Kirkil G, Lower EE, Baughman RP. Predictors of mortality in pulmonary sarcoidosis. Chest 2018; 153: 105-113. 
19 Drent M, De Vries J, Lenters M, et al. Sarcoidosis: assessment of disease severity using HRCT. Eur Radiol 2003; 13: 2462-2471.

20 Benamore R, Kendrick YR, Repapi E, et al. CTAS: a CT score to quantify disease activity in pulmonary sarcoidosis. Thorax 2016; 71: 1161-1163.

21 Keijsers RG, Verzijlbergen EJ, van den Bosch JM, et al. 18F-FDG PET as a predictor of pulmonary function in sarcoidosis. Sarcoidosis Vasc Diffuse Lung Dis 2011; 28: 123-129.

22 Cao Z, Zhu W, Wang W, et al. Stable cerasomes for simultaneous drug delivery and magnetic resonance imaging Int J Nanomed 2014; 9: 5103-5116.

23 Stephen MJ, Emami K, Woodburn JM, et al. Quantitative assessment of lung ventilation and microstructure in an animal model of idiopathic pulmonary fibrosis using hyperpolarized gas MRI. Acad Radiol 2010; 17 : $1433-1443$.

24 Schupp JC, Freitag-Wolf S, Bargagli E, et al. Phenotypes of organ involvement in sarcoidosis. Eur Respir J 2018; 51: 1700991 .

25 Kataria YP, Whitcomb ME. Splenomegaly in sarcoidosis. Arch Intern Med 1980; 140: 35-37.

26 Rybicki BA, Sinha R, Iyengar S, et al. Genetic linkage analysis of sarcoidosis phenotypes: the sarcoidosis genetic analysis (SAGA) study. Genes Immun 2007; 8: 379-386.

27 James DG, Sharma OP. Neurosarcoidosis. Proc R Soc Med 1967; 60: 1169-1170.

28 Lower EE, Broderick JP, Brott TG, et al. Diagnosis and management of neurological sarcoidosis. Arch Intern Med 1997; 157: 1864-1868.

29 James DG, Neville E, Siltzbach LE. A worldwide review of sarcoidosis. Ann N Y Acad Sci 1976; 278: 321-334

30 Pietinalho A, Ohmichi M, Hiraga Y, et al. The mode of presentation of sarcoidosis in Finland and Hokkaido, Japan. A comparative analysis of 571 Finnish and 686 Japanese patients. Sarcoidosis Vasc Diffuse Lung Dis 1996; 13: 159-166.

31 Baughman RP, Teirstein AS, Judson MA, et al. Clinical characteristics of patients in a case control study of sarcoidosis. Am J Respir Crit Care Med 2001; 164: 1885-1889.

32 Schulze TG, McMahon FJ. Defining the phenotype in human genetic studies: forward genetics and reverse phenotyping. Hum Hered 2004; 58: 131-138.

33 Iannuzzi MC, Baughman RP. Reverse phenotyping in sarcoidosis. Am J Respir Crit Care Med 2007; 175: 4-5.

34 Wijsenbeek MS, Culver DA. Treatment of sarcoidosis. Clin Chest Med 2015; 36: 751-767.

35 Culver DA. Diagnosing sarcoidosis. Curr Opin Pulm Med 2015; 21: 499-509.

36 Patel MR, Cawley PJ, Heitner JF, et al. Detection of myocardial damage in patients with sarcoidosis. Circulation 2009; 120: 1969-1977.

37 Iwai K, Tachibana T, Takemura T, et al. Pathological studies on sarcoidosis autopsy. I. Epidemiological features of 320 cases in Japan. Acta Pathol Jpn 1993; 43: 372-376.

38 Hercules HD, Bethlem NM. Value of liver biopsy in sarcoidosis. Arch Pathol Lab Med 1984; 108: 831-834.

39 Selroos O. Sarcoidosis of the spleen. Acta Med Scand 1976; 200: 337-340.

40 Wallace SL, Lattes R, Malia JP, et al. Muscle involvement in Boeck's sarcoid. Ann Intern Med 1958; 48: 497-511.

41 Judson MA. The three tiers of screening for sarcoidosis organ involvement. Respir Med 2016; 113: 42-49.

42 Hunninghake GW, Costabel U, Ando M, et al. ATS/ERS/WASOG statement on sarcoidosis. American Thoracic Society/European Respiratory Society/World Association of Sarcoidosis and other Granulomatous Disorders. Sarcoidosis Vasc Diffuse Lung Dis 1999; 16: 149-173.

43 Birnie DH, Sauer WH, Bogun F, et al. HRS expert consensus statement on the diagnosis and management of arrhythmias associated with cardiac sarcoidosis. Heart Rhythm 2014; 11: 1305-1323.

44 Judson MA, Baughman RP, Thompson BW, et al. Two year prognosis of sarcoidosis: the ACCESS experience. Sarcoidosis Vasc Diffuse Lung Dis 2003; 20: 204-211.

45 Inoue $\mathrm{Y}$, Inui $\mathrm{N}$, Hashimoto $\mathrm{D}$, et al. Cumulative incidence and predictors of progression in corticosteroid-naive patients with sarcoidosis. PLoS One 2015; 10: e0143371. 